\title{
ASSESSMENT OF ANALGESIC EFFECT OF BREAST MILK, ACETAMINOPHEN AND NO INTERVENTION FOR TERM NEONATES UNDERGOING PERCUTANEOUS VENOUS CATHETER PLACEMENT AND REPLACEMENT
}

\section{A. Mortazavi}

Social Security Organizaation Hospital, Zandjan, Iran

Background and aims: All researchers according to AAP comments for neonatal pain treatment try to find low risk and safe therapies. So our study compares analgesic effects of breast milk and acetaminophen with each other and control group (no analgesic administration before percutaneous venous catheter placement and replacement).

Methods: This interventional experimental random clinical trial study was accomplished in over one year from February 2009 to February 2010 on180term, stable admitted neonates. The Neonatal Infant Pain Scale (NIPS) and CRIES pain measurement score were accepted for determine and comparison of pain scales in our samples (Table-1). Research population divided to A (acetaminophen usage), B (breast milk feeding) and $\mathrm{C}$ (control) group equally with simple random method. For all groups total mean pain score calculated (table$2)$. Data were analyzed using SPSS statistical software and Paired t-tests and tukey test. $P$-value less than 0.05 were considered significant.

Results: Mean neonatal pain score of breast feeding group population (B) was $2.18(\mathrm{SD}=2.7)$ approximately 2. 75 times lower than acetaminophen using group (A) and 3.5 times lower than control group (C). The difference of total mean pain score of each group (Fig-2), compared with tukey test. Differences were significant $(\mathrm{p}=0.001)$.Comparing of mean total pain score of A group [total mean pain score $=7.6(\mathrm{SD}=5.6)$ ] and $\mathrm{C}$ group [total mean pain score $=6(\mathrm{SD}=5)$ ] showed that $\mathrm{A}$ group had higher total mean pain score probably due to bitter taste of acetaminophen and delay analgesic effect of acetaminophen.

Conclusion: Breast milk has analgesic effect before percutaneous venous catheter placement / replacement.

\begin{tabular}{|l|l|l|l|l||l|}
\hline signs/scale & 0 & 1 & 2 & 3 \\
\hline \hline Brow bulges & NO & $<1$ minute & $1-2$ minutes & $2-3$ minutes & $>4$ minutes \\
\hline Eye squeeze & NO & $<1$ minute & $1-2$ minutes & $2-3$ minutes & $>4$ minutes \\
\hline \hline Crying & NO & $<1$ minute & $1-2$ minutes & $2-3$ minutes & $>4$ minutes \\
\hline Body movement & NO & $<1$ minute & $1-2$ minutes & $2-3$ minutes & $>4$ minutes \\
\hline Heart rate & $<180 / \mathrm{min}$ & $181-185 / \mathrm{min}$ & $186-190 / \mathrm{min}$ & $191-195 / \mathrm{min}$ & $>195 / \mathrm{min}$ \\
\hline o2 saturation & $>95 \%$ & $90-94 \%$ & $85-89 \%$ & $80-84 \%$ & $<80 \%$ \\
\hline
\end{tabular}

[Pain measurement score]

\begin{tabular}{|l|l|l|l|l|}
\hline & Pain Score & {$[$ Mean $(\mathrm{SD}]$} & & \\
\hline \hline & Girls & Boys & P value & Total score \\
\hline A group & $8.1(5.6)$ & $7.2(5.6)$ & 0.55 & $7.6(5.6)$ \\
\hline B group & $2.27(3.2)$ & $2.13(2.4)$ & 0.96 & $2.18(2.7$ \\
\hline C group & $7.8(5.8)$ & $5.2(4.4)$ & 0.2 & $6(5)$ \\
\hline
\end{tabular}

[Neonatal pain scores in case (A and $B$ ) groups and]



[Fig-1: Total Mean pain scores of case and control groups] 